\title{
THE EFFECT OF SYNTHETIC PATCH REPAIR OF COARCTATION ON REGIONAL DEFORMATION OF THE AORTIC WALL
}

Bruce H. Smaill, $\mathrm{PhD}^{\mathrm{a}}$

David C. McGiffin, MB, BS, FRACS ${ }^{b}$

Ian J. LeGrice, MB, BS, PhD

Alistair A. Young, $\mathrm{PhD}^{\mathrm{a}}$

Peter J. Hunter, DPhil ${ }^{\mathrm{c}}$

Andrew J. Galbraith, BMedSc, MB, BS, FRACPd
Background: A long-term complication of synthetic patch repair of coarctation is true aneurysm formation.

Aim: An in vitro study was undertaken to determine the effects of patch angioplasty on aortic geometry and strain adjacent to the patch.

Methods: Segments of human descending thoracic aorta were subject to 10 pressure loading cycles (10-120 mm Hg; 1.36-16.32 $\mathrm{kPa}$ ) before and after simulated coarctation repair with a synthetic patch. Local curvature and strain were estimated by fitting a geometric model to reconstructed threedimensional surface marker points.

Results: In the control aortas, when pressure increased from $11 \pm 1.0$ to 124 $\pm 4.0 \mathrm{~mm} \mathrm{Hg}(1.5 \pm 0.14$ to $16.86 \pm 0.54 \mathrm{kPa})$, average circumferential curvature decreased from $0.1543 \pm 0.03$ to $0.1065 \pm 0.03 \mathrm{~mm}^{-1}$. The average major extension reached a maximum of $1.43 \pm 0.08$. After patch implantation, the average circumferential curvature was reduced relative to control at all pressures. Average major extensions were significantly greater than paired control values and reached a maximum of $1.55 \pm 0.08$ at $122 \pm 4.0$ $\mathrm{mm} \mathrm{Hg}(16.59 \pm 0.54 \mathrm{kPa})$. Substantial strain inhomogeneity was observed and major extensions were greatest immediately adjacent to the patch.

Inference: Synthetic patch repair of coarctation of the aorta increases wall strain and produces significant regional gradients in strain. With control aortic material properties there may be a substantial increase in wall stress immediately adjacent to the aorta, which could lead to true aneurysm formation. (J Thorac Cardiovasc Surg 2000;120:1053-63)
$\mathrm{R}$ epair of coarctation of the aorta by synthetic patch aortoplasty was first described by Vosschulte $^{1}$ in 1957. This technique is an effective means of relieving the stenosis, with the advantage that subsequent growth

From the Departments of Physiology ${ }^{\mathrm{a}}$ and Engineering Science, ${ }^{\mathrm{c}}$ University of Auckland, Auckland, New Zealand; Department of Surgery, ${ }^{\mathrm{b}}$ University of Alabama at Birmingham, Birmingham, Ala; and the Department of Cardiology, ${ }^{\mathrm{d}}$ The Prince Charles Hospital, Brisbane, Australia.

Supported by a grant from the National Heart Foundation of Australia (grant No. G89B2741).

Received for publication Feb 17, 2000; revisions requested April 26, 2000; revisions received July 13, 2000; accepted for publication July 18, 2000.

Address for reprints: David McGiffin, MD, Department of Surgery, Division of Cardiothoracic Surgery, The University of Alabama at Birmingham, 780 Lyons-Harrison Research Building, 1919 Seventh Ave South, Birmingham, AL 35294-0007.

Copyright (C) 2000 by The American Association for Thoracic Surgery

$0022-5223 / 2000 \$ 12.00+0 \quad \mathbf{1 2 / 1 / 1 1 0 1 8 7}$

doi:10.1067/mtc.2000.110187 of the aorta in the region of the repair is possible. However, a high incidence of aneurysm formation at the site of the repair has been reported. ${ }^{1-17}$ The characteristics of these aneurysms include the fact that they are true aneurysms, as opposed to false aneurysms (which occur at the suture line), that they arise from the aortic wall opposite the patch, $, 2,8$ and that they are associated with fragmentation or loss of elastin layers within the aneurysm and in regions adjacent to the patch. 5,7,8,16

Reasons advanced for the development of aneurysms include the disparate elastic properties of the aorta and synthetic patch ${ }^{2}$ and resection of the posterior intimal shelf. ${ }^{9}$ On the other hand, it has been argued that fragmentation or loss of elastin layers in the peripatch region may reflect underlying tissue abnormality associated with the initial coarctation of the aorta rather than the repair. ${ }^{4}$

A study of synthetic patch repair of coarctation by McGiffin and colleagues ${ }^{18}$ used finite element analysis to examine aortic wall stress at the site of the repair. 
Aortic wall stress at the site of repair was shown to increase out of proportion to the increase in diameter of the aorta because of placement of the synthetic patch. Although this study was not designed to relate increases in aortic wall stress to subsequent aneurysm formation, the abnormally high aortic wall stress predicted opposite the patch suggests that the aorta may be operating close to its strain limit in this region. This would predispose the aorta to structural change that would likely result in elastin fiber fracturing and degeneration.

Important limitations of this analysis are that nonlinear effects were not considered in a full three-dimensional (3-D) analysis and that it did not yield information on the regional distribution of strain in the aorta. There is an evident requirement for a carefully controlled experimental study of the biomechanical effects of synthetic patch repair of aortic coarctation by using human tissue. It is important to note that whereas strain and deformation can be estimated in soft tissue, stress cannot be reliably measured.

This study was undertaken to characterize the distribution of strain in the aortic wall adjacent to a synthetic patch to test the hypothesis that patch repair of coarctation leads to substantial increases on aortic strain (and stress).

\section{Materials and methods}

The objective of this study was to characterize the changes in geometry and strain in the region adjacent to a patch enlargement of the aortic wall. The experiment was not intended to simulate the conditions of coarctation repair. However, enlargement of the descending thoracic aorta by a patch, which is the inevitable consequence of repairing a coarctation by the patch angioplasty technique, was replicated.

Tissue procurement. Measurements were made in segments of human descending thoracic aorta. The tissue was obtained at the time of autopsy of trauma victims at the Queensland Government Institute of Forensic Pathology, Brisbane, Australia. Permission was obtained from relatives of the victims for removal of tissue as part of a general consent for use of allograft valves and tissue for scientific investigation. Ethical approval for the project was granted by the Ethics Committee of the Prince Charles Hospital, Brisbane, Australia. After procurement, the segments of descending thoracic aorta were trimmed of areolar tissue, packaged in nutrient medium (Medium 199; Commonwealth Serum Laboratories, Parkville, Victoria, Australia) and frozen at $0^{\circ} \mathrm{C}$. It has previously been demonstrated that storage of aortic tissue by freezing in saline solution does not alter the material properties of the tissue. ${ }^{19}$ Aorta was procured from 7 victims of trauma (4 male and 3 female), ages ranging from 9 to 25 years.

Tissue preparation. Frozen segments of aorta were removed from the packaging and thawed. An inflatable internal sleeve was formed by drawing a thin latex tube through the aorta and securing the aorta and lining at each end to a cannula (Fig 1). Circumferential columns of fine India ink dots (2-3 mm apart) were then marked on the adventitia of the aorta. A 7-0 polypropylene suture was then sutured to the adventitia so that it lay longitudinally on the posterior aspects of the aorta. This was used as a landmark during marker coordinate reconstruction.

Recording system. The aorta was mounted at the center of a $45^{\circ}$ biplane video camera arrangement (Fig 2). The blindended lower cannula was attached to a base plate. The upper cannula was supported by a rigid bar and connected to a volume infusion pump (Harvard Apparatus Co, Inc, S Natick, Mass), enabling the aorta to be inflated (and deflated) with saline solution at a controlled rate. Pressure in the aorta was monitored by a strain gauge transducer (Statham P23DB; AMETEK, Inc, Paoli, Pa) via a port in the lower cannula.

The 2 cameras (Ikegama ICD-42E fitted with macro lenses; Ikegama Laboratory, Tokyo, Japan) were mounted on linear positioning racks. The video cameras were connected via a frame grabber (Matrox PIP 1024; Matrox Electronic Systems Ltd, Dorval, QC, Canada) to a videocassette recorder. Positions of the cameras and aortic pressure were monitored continuously at $50 \mathrm{~Hz}$ by means of a Tandon 386 microcomputer (Tandon Corporation, Moorpark, Calif) that also controlled the frame grabber. The computer was programmed to acquire these data (sampling interval 2 seconds) on operator command and simultaneously to switch the camera inputs to the frame grabber so that sequential biplane views of the aorta could be recorded. The sample number was overlaid on the video record.

Experimental protocol. The aorta was prestressed slightly to minimize buckling during inflation. A pressure of 60 $\mathrm{mm} \mathrm{Hg}(8.16 \mathrm{kPa})$ was applied and the axial position of the upper cannula was adjusted until the aorta was cylindrical. The aorta was then subjected to 10 cycles of volume infusion and withdrawal at a fixed rate over a pressure range 10 to 120 $\mathrm{mm} \mathrm{Hg}$ (1.36-16.32 kPa). The cycle duration (1-2 minutes) was fixed for each experiment. Pressure, camera positions, and biplane video images were acquired continuously.

When this control sequence was complete, the aorta and cannulas were removed from the recording system. A longitudinal incision was made in the anterior wall of the aorta, and an oval patch of Dacron graft was sutured to the aortotomy. Synthetic patches were fashioned with different dimensions to produce degrees of patch ballooning of the extent typically seen in surgical practice. Synthetic patch length ranged from $11.2 \mathrm{~mm}$ to $27.4 \mathrm{~mm}$ and patch width ranged from $8.5 \mathrm{~mm}$ to $20.4 \mathrm{~mm}$. All grafts were sutured with $4-0$ polypropylene. The aorta was then remounted in the recording system and the full sequence used for control testing was repeated.

On completion of the experiment, the aorta was removed and filled with silicone rubber molding material (Dow Corning RTV; Dow Corning, Midland, Mich) for subsequent determination of geometry. Dimensions were obtained for each specimen and key measures are presented in Table I.

Marker coordinate reconstruction. Recorded images of the aorta were digitized and displayed on a color video monitor so that corresponding marker points in both views could 


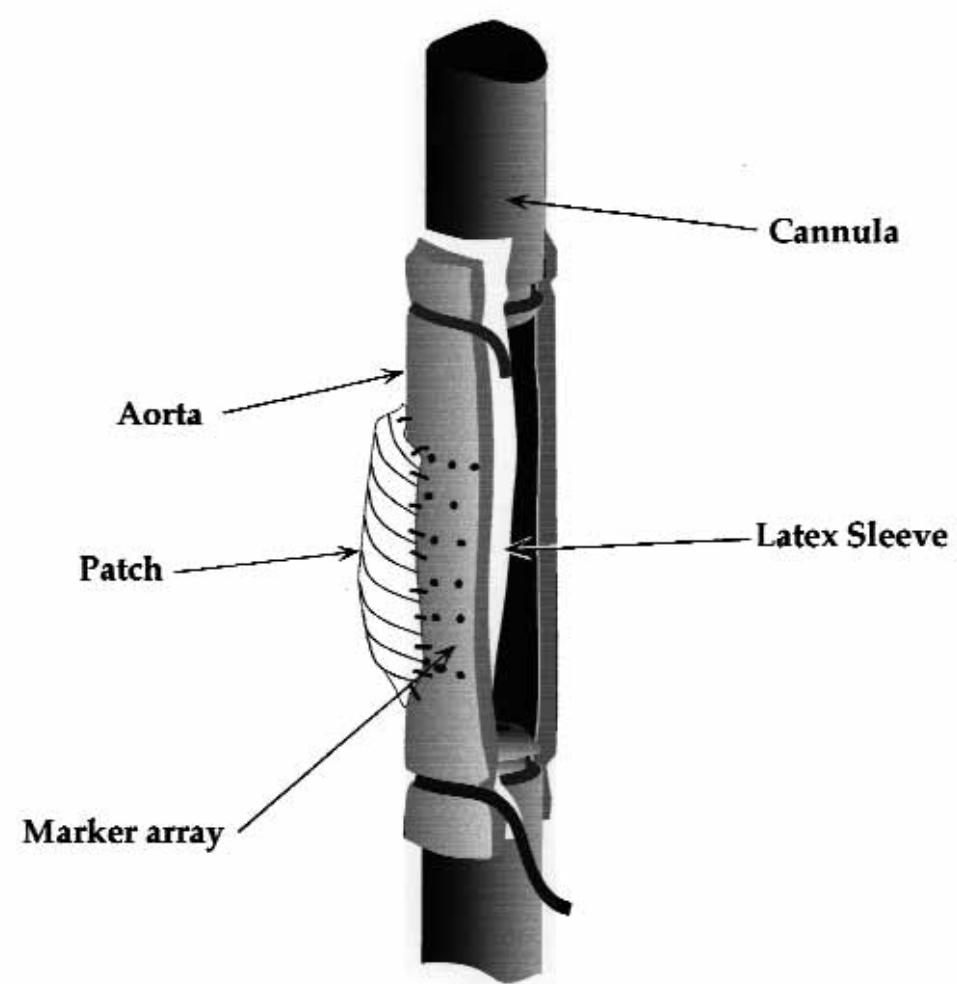

Fig 1. Diagram illustrating the way in which specimens of aorta were mounted for testing. The specimen and the compliant internal latex sleeve are secured to a cannula at each end. A synthetic patch is sutured to the aorta and a part of the matrix of marker points can be seen.

be readily identified. A computer mouse was used to overlay fine crosses on associated points in both views. The spatial coordinates for each point were determined from their screen coordinates in the 2 camera views by means of methods outlined elsewhere. ${ }^{20}$ Coordinates of the lateral boundaries of the aorta, at corresponding vertical positions in both camera views, were also estimated in a similar fashion.

Marker positions were reconstructed for the infusion phase of the ninth or tenth loading cycle. Between 27 and 51 points were tracked for the samples at pressures that were closest to 10, 20, 40, 60, 80, 100, and $120 \mathrm{~mm} \mathrm{Hg}(1.36,2.72,5.44$, $8.16,10.88,13.6$, and $16.32 \mathrm{kPa}$ ).

Lateral boundary coordinates at these pressures were estimated at 2 positions below and/or above the patch and at 6 to 8 vertical positions for the control sequence. The coordinates of points along the edge of the patch were also reconstructed at the reference pressure (close to $10 \mathrm{~mm} \mathrm{Hg} ; 1.36 \mathrm{kPa}$ ).

The relationship of the 3-D coordinates of any marker point to its screen coordinates in biplane camera views depends on the distortion introduced by the lenses, perspective effects due to displacement of the point from the cameras, the angle of intersection of camera axes, and the distance of the cameras from the intersection point. Distortion introduced by the macro lenses in our system occurs at the periphery of the image $^{20}$ and can be neglected for the fields used in this study. Because biplane angle and camera position are known, it is necessary only to identify the linear perspective transforma-
Table I. Dimensions of aorta and patch

\begin{tabular}{cccc}
\hline Experiment & $\begin{array}{c}\text { Diameter of } \\
\text { aorta }^{*}(\mathrm{~mm})\end{array}$ & $\begin{array}{c}\text { Diameter of } \\
\text { repair }(\mathrm{mm})\end{array}$ & Patch ratio \\
\hline 1 & 11.1 & 18.2 & 1.6 \\
2 & 13.2 & 22.1 & 1.7 \\
3 & 12.4 & 12.4 & 1.0 \\
4 & 11.2 & 17.1 & 1.5 \\
5 & 14.8 & 22.0 & 1.5 \\
6 & 13.2 & 18.5 & 1.4 \\
\hline
\end{tabular}

Measurements could not be obtained for experiment 7 because of failure of the molding process.

*Because of a small degree of distortion produced by the molding process, the aortic diameter is the average of the diameter measured in 2 planes at right angles.

tions. To do this, a calibration grid $(200 \times 300 \mathrm{~mm}$ with $1-\mathrm{mm}$ ruling and cumulative accuracy better than $0.02 \mathrm{~mm}$ ) was placed perpendicular to camera 1 at the center of the recording system and recorded for both cameras over their full range of positions. In each view, selected points were tracked for camera positions spanning $250 \mathrm{~mm}$ and parameters were fitted by the least squares method.

The accuracy of coordinate reconstruction was checked by comparing actual and estimated coordinates of selected points in records of the calibration grid. This procedure was 


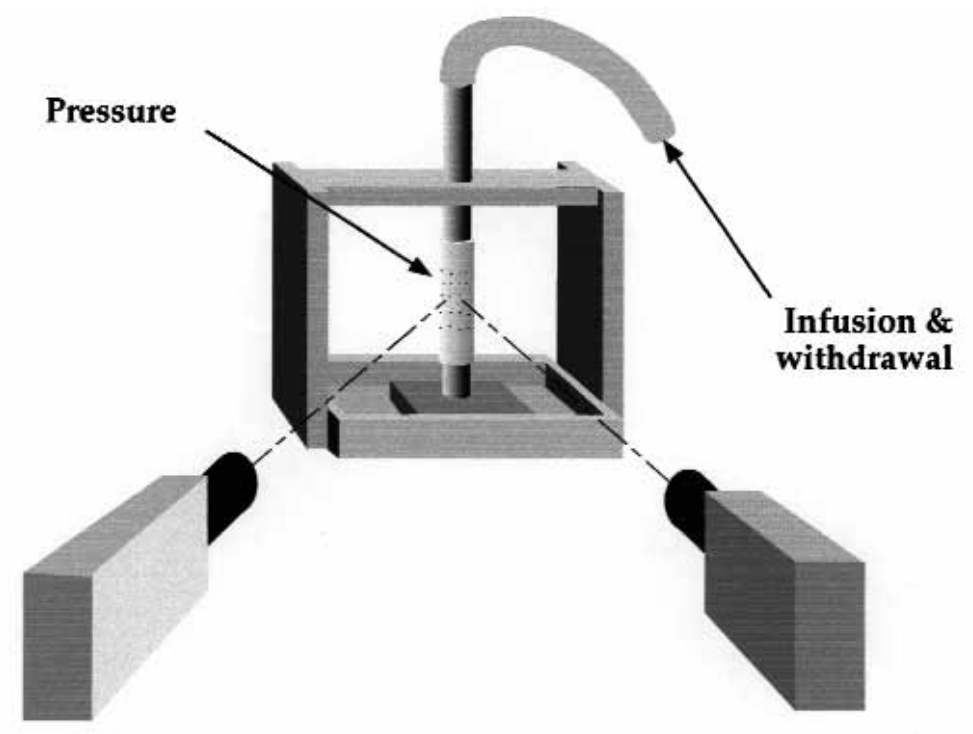

Video Camera 2

Video Camera 1

Fig 2. Schematic representation of the biplane video imaging system, vertically mounted specimen of aorta, and pressurization system.

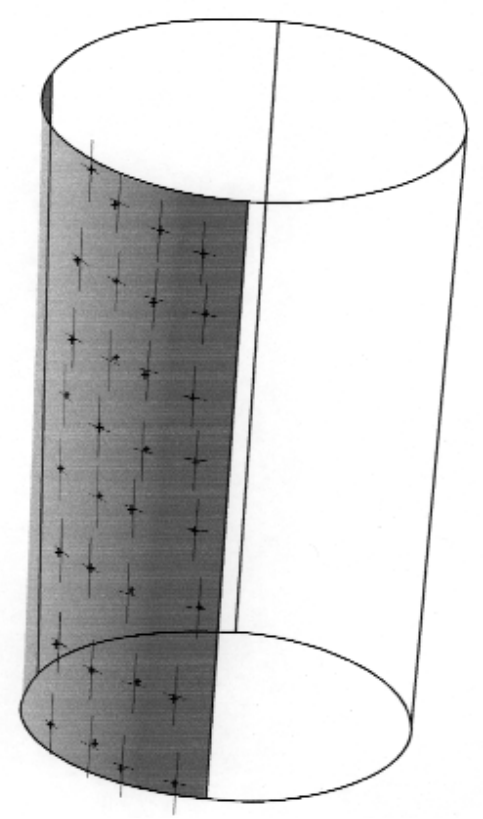

Fig 3. Initially cylindrical finite element mesh fitted to the 3D coordinates of aortic marker points. The small crosses represent reconstructed marker points from aorta \#1 in unpatched reference state $(11.2 \mathrm{~mm} \mathrm{Hg} ; 1.52 \mathrm{kPa})$. Normal projections from the shaded surface to these points indicate the residual error between the surface and marker points. The finite element surface was fitted by minimizing an error function that includes the squared sum of residuals. The rms error is $0.14 \mathrm{~mm}$. carried out on several occasions for up to 25 uniformly distributed points in the range $\mathrm{x}= \pm 10 \mathrm{~mm}, \mathrm{y}=0 \mathrm{~mm}, \mathrm{z}= \pm 10$ $\mathrm{mm}$. Mean reconstruction accuracy for each coordinate was $\mathrm{x}=0.002 \pm 0.074 \mathrm{~mm}, \mathrm{y}=-0.008 \pm 0.096 \mathrm{~mm}, \mathrm{z}=0.039 \pm$ $0.055 \mathrm{~mm}$. Maximum deviations were $\mathrm{x}=-0.2 \mathrm{~mm}, \mathrm{y}=0.3$ $\mathrm{mm}, \mathrm{z}=-0.2 \mathrm{~mm}$ and in all cases these occurred at the periphery of the field.

Reconstruction of aortic geometry. The geometry of the aorta was reconstructed by fitting a finite element surface to the marker points (Fig 3). The aorta was modeled as an initially cylindrical finite element mesh consisting of 4 surface elements. Marker points were converted from Cartesian to cylindrical polar coordinates $(\mathrm{r}, \phi, \mathrm{z})$ and the element boundaries were defined so that data points were contained in a single element (element 1), with the remaining elements evenly subdivided circumferentially. The interpolating basis functions for each of the 3 surface coordinates were bicubic Hermite polynomial. The error function minimized in the fitting process consisted of 2 terms. These were, first, the sum of squared residuals between data points and corresponding points on the fitted surface and, second, a quadratic smoothing term that penalized first and second derivatives of the displacement from initial mesh locations-for a more detailed account, see Young and associates. ${ }^{21}$ The use of this smoothing penalty, together with cylindrical topology, constrained the fitted surface at the boundaries of element 1 .

For both control and patched cases, the mesh was initially fitted in the radial coordinate only at the reference state (close to $10 \mathrm{~mm} \mathrm{Hg}$ ). For subsequent steps in control and patched loading cycles, the mesh was deformed to match the motion of the material points and deformed surfaces were fitted in all 3 coordinates (Fig 4). Smoothing weights were adjusted to 


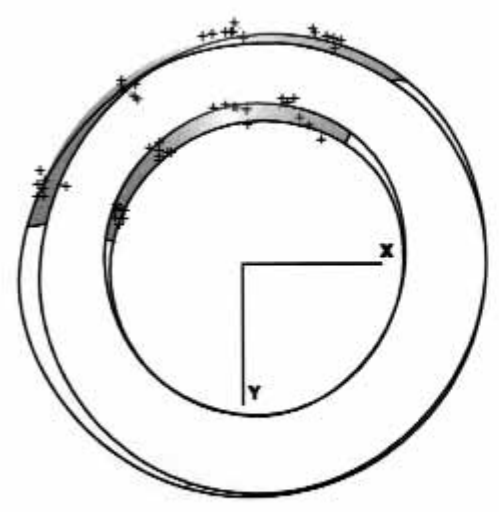

a) b)

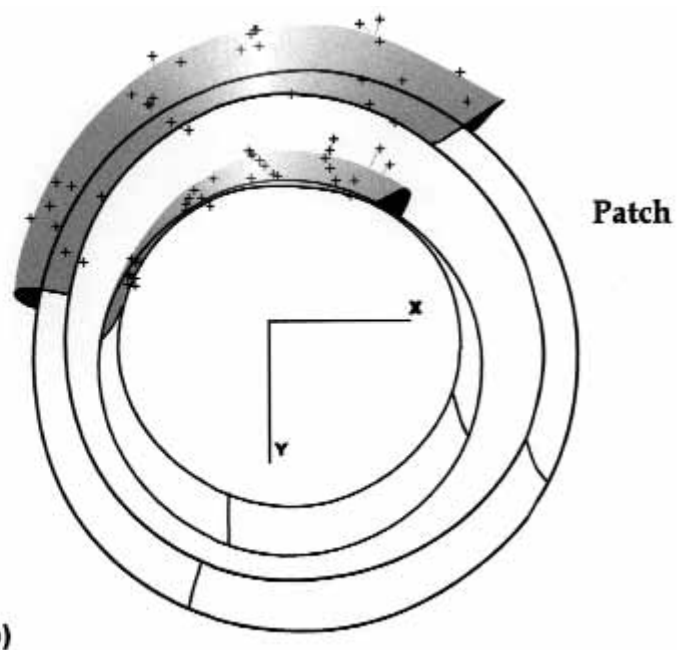

Fig 4. Finite element surfaces fitted to reconstructed marker points (small crosses) from aorta \#6 viewed from above. a, Control: The inner reference data set was obtained at a pressure of $11.0 \mathrm{~mm} \mathrm{Hg}(1.5 \mathrm{kPa})$, and the outer data set was obtained at $122.5 \mathrm{~mm} \mathrm{Hg}(16.66 \mathrm{kPa})$. The rms errors for reference and deformed surfaces are 0.23 $\mathrm{mm}$ and $0.22 \mathrm{~mm}$, respectively. b, Patched: The inner reference data set was obtained at a pressure of $9.7 \mathrm{~mm} \mathrm{Hg}$ $(1.32 \mathrm{kPa})$, and the outer data set was obtained at $125.9 \mathrm{~mm} \mathrm{Hg}(17.12 \mathrm{kPa})$. The rms errors for reference and deformed surfaces are $0.42 \mathrm{~mm}$ and $0.44 \mathrm{~mm}$, respectively. The patch was immediately to the right of the shaded surface.

produce root mean squared (rms) errors for the control reference state on the order of the measurement error. The same weights were used for control and patched loading cycles.

Determination of aortic curvature. Surface curvature was characterized by using techniques outlined in Young and associates. ${ }^{21}$ Circumferential and longitudinal curvatures were estimated for the 9 gaussian quadrature points in element 1 , together with principal curvatures.

Estimation of regional strain. The Green strain tensor associated with the deformation of the finite element mesh was estimated at the 9 quadrature points in element 1 . The surface strain components $\mathrm{E}_{\phi \phi}, \mathrm{E}_{\mathrm{zz}}, \mathrm{E}_{\phi \mathrm{z}}$ (circumferential, longitudinal and longitudinal-circumferential strain, respectively) were also decomposed into the principal ${ }^{*}$ strains $E_{1}, E_{2}$, referred to as major and minor strains, respectively. In this article, $\mathrm{E}_{\mathrm{\phi}}, \mathrm{E}_{\mathrm{zz}}, \mathrm{E}_{1}$, and $\mathrm{E}_{2}$ are converted to extension ratios by using the relationship $\mathrm{E}=0.5\left(\lambda^{2}-1\right)$, where $\lambda$ is the extension ratio.

Strain was determined with respect to separate reference states for control and patched cases, because the sets of marker points imaged in the control study were not identical to those observed after patch placement. Under these circum-

\footnotetext{
*The surface strain at any point is characterized by specifiying the 2 principal strains $E_{1}$ and $E_{2}$ and their orientations. $E_{1}$ and $E_{2}$ are plane (tensile or compressive) strains and are orthogonal. The major strain $\mathrm{E}_{1}$ is the maximum strain at the point, the minor strain is the minimum strain, whereas the principal angle specifies the orientation of $\mathrm{E}_{1}$ with respect to the circumferential direction. Principal curvatures may be interpreted in the same fashion.
}

stances, the strain analysis for the patched state does not reflect the residual deformation due to patch placement. To address this problem, we identified a subset of common material points in both control and patched states, and the initial strain in the patched reference state was estimated. In each experiment, deformation gradient tensors estimated for this state were used to correct the strain determined for the patched state.

Data analysis. All data are expressed as mean \pm SD. Comparison of grouped data was made by multiway repeated measures analysis of variance (ANOVA).

\section{Results}

General. Reproducible aortic pressure-volume cycles with moderate hysteresis were observed after the second cycle of inflation and deflation for control and patched states. The coordinate analysis carried out for the inflation phase of the ninth or tenth cycles for each experiment therefore provided representative data.

The precision with which the aortic surface was reconstructed in both control and patched states was wholly acceptable. Root mean squared (rms) errors for the finite element surface fits to 3-D marker coordinates ranged from $0.02 \mathrm{~mm}$ to $0.44 \mathrm{~mm}$ with a mean value of $0.13 \mathrm{~mm}$. Fig 3 presents the fitted reference surface for a typical control experiment (rms error $0.138 \mathrm{~mm}$ ). In Fig 4, fitted reference surfaces are compared with deformed surfaces for $(a)$ control and $(b)$ patched cases. Fig 4, $a$, demonstrates that, before patch 


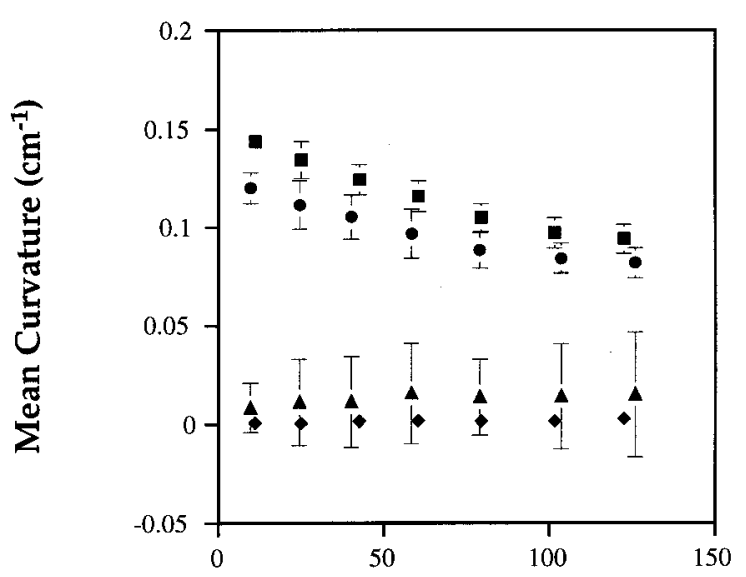

(a)

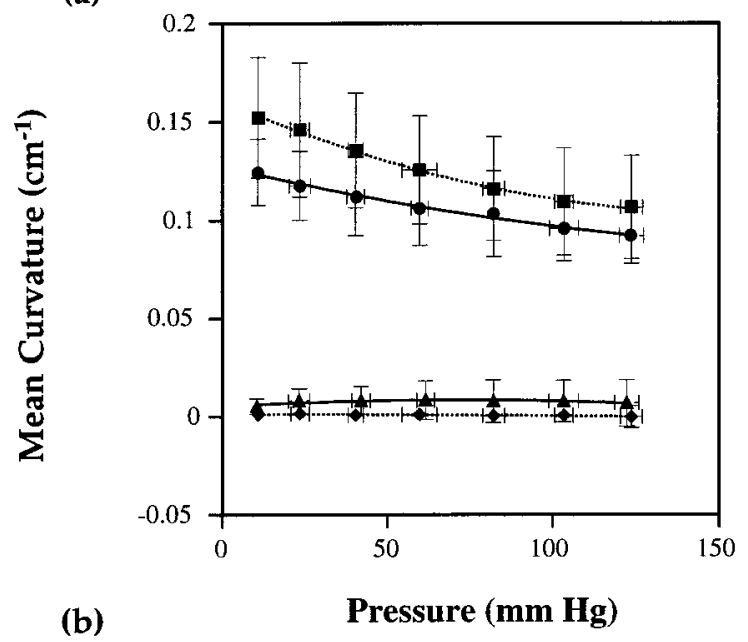

Fig 5. Circumferential and longitudinal curvatures as a function of pressure: $\mathbf{c}$ control circumferential curvature; circumferential curvature with patch; control longitudinal curvature; $\boldsymbol{\Delta}$ longitudinal curvature with patch. a, Mean curvatures for all 9 gauss quadrature points in aorta \#6. Error bars indicate standard deviations for curvature. b, Grand mean for all studies. Error bars indicate standard deviations for curvature and pressure.

placement, marker points in reference and inflated states are well fit by a cylindrical surface patch. This was a consistent result for all control experiments. Patch angioplasty distorted the aorta adjacent to patch (compare reference states for Figs 4, $a$ and $b$ ), and the surfaces fitted at all levels of inflation were more complex than for corresponding control results. Root mean squared (rms) errors were greater in the patched than in the control case. Errors typically increased adjacent to the patch (Fig 4, $b$, is the worst-case result).

Curvature. Principal (maximum and minimum) curvatures coincided with circumferential and longitudinal
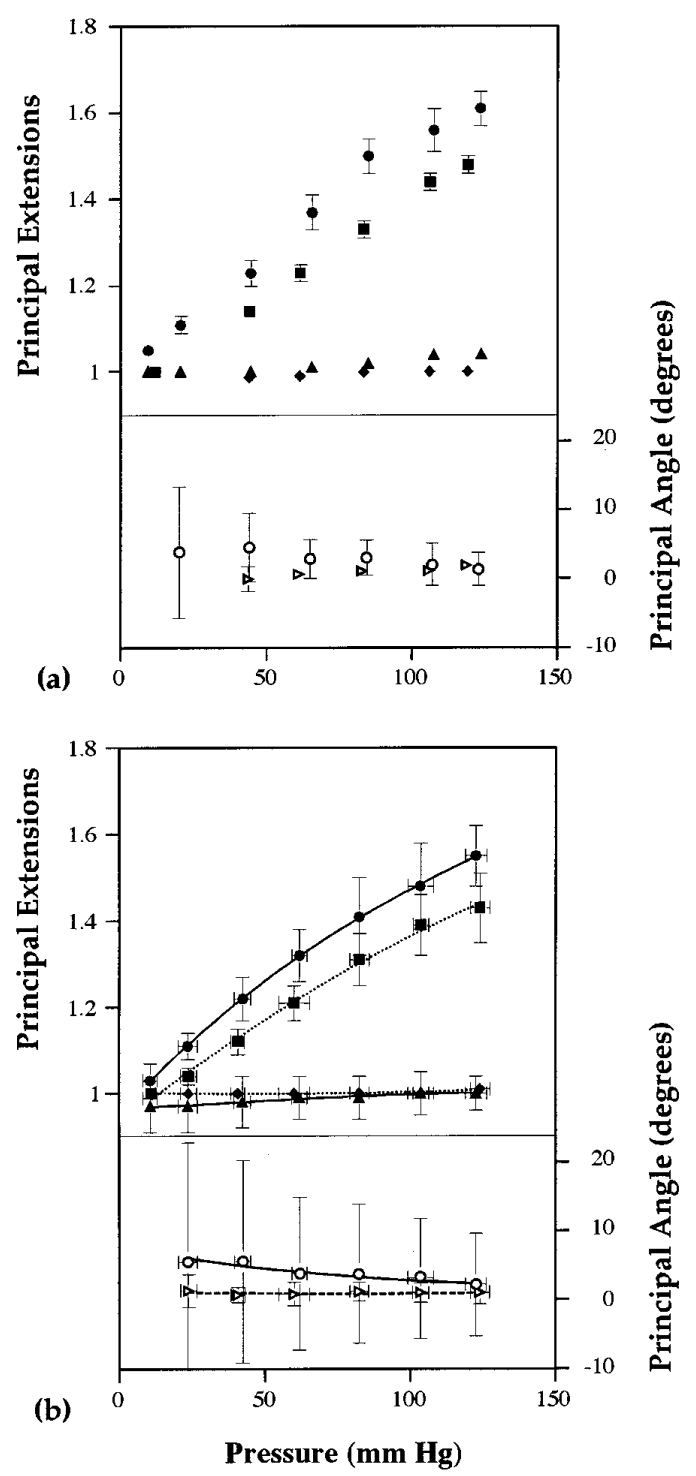

Fig 6. First principal (major) and second principal (minor) extensions as a function of pressure: control major extension; major extension with patch; $\bullet$ control minor extension; $\Delta$ minor extension with patch; $\triangle$ control principal angle; $\bigcirc$ principal angle with patch. a, Mean principal extensions for all 9 gauss quadrature points in aorta \#3. b, Grand mean for all studies. Error bars indicate standard deviations for principal extensions and pressure.

directions for both control and patched states. Fig 5, $a$, presents data for a typical experiment. In the control state there was a progressive reduction in mean circumferential curvature with increased pressure, whereas longitudinal curvature was unchanged. There was little variation in circumferential and longitudinal curvature over the 9 gauss points in element 1 for the control case. Overall, 
Table II. Extensions in aorta adjacent to patch in unloaded state

\begin{tabular}{|c|c|c|c|c|c|c|c|c|c|}
\hline & \multicolumn{7}{|c|}{ Experiment } & \multirow[b]{2}{*}{ Mean } & \multirow[b]{2}{*}{$\mathrm{P}$ value } \\
\hline & 1 & 2 & 3 & 4 & 5 & 6 & 7 & & \\
\hline$\lambda_{\phi \phi}$ & 1.04 & 1.03 & 1.04 & 1.01 & 1.05 & 1.07 & 0.95 & $1.03 \pm 0.04$ & $.05^{1}$ \\
\hline$\lambda_{\mathrm{zz}}^{\phi \phi}$ & 0.95 & 0.95 & 1.00 & 0.95 & 1.01 & 0.88 & 1.08 & $0.97 \pm 0.06$ & $.2^{1}$ \\
\hline$\gamma_{\mathrm{dz}}$ & -0.18 & -0.62 & -0.42 & 4.53 & -0.95 & 0.93 & 1.30 & $0.66 \pm 1.90$ & $.2^{2}$ \\
\hline
\end{tabular}

$\lambda_{\phi \phi} \& \lambda_{\mathrm{zz}}$ are circumferential and longitudinal extension ratios, respectively. $\gamma_{\phi \mathrm{z}}$ is the $\phi$-z shear angle in degrees. Extensions are determined from the deformation gradients in unloaded state after patch angioplasty with respect to unloaded control state.

1. Unpaired $t$ test of hypotheses that $\lambda_{\phi \phi}$ and $\lambda_{z z}$ differ from 1.0 (1-tailed distribution).

2. Unpaired $t$ test of hypotheses that the $\gamma_{\phi z}$ differs from zero (2-tailed distribution).

these results are entirely consistent with the inflation of a cylinder. Circumferential curvature was reduced for all pressures in the patched state, indicating that patch angioplasty flattens the adjacent aorta. On the other hand, there appears to be a small increase in longitudinal curvature in the patched case consistent with ballooning of the aorta immediately adjacent to the patch. The widened standard deviations indicate that patch angioplasty produces substantial regional variation of curvature in the region adjacent to the patch.

Average results presented in Fig 5, $b$, are congruent across all studies. Whereas circumferential curvature decreases with pressure in both control and patched cases, patch implantation reduces circumferential curvature with respect to paired controls at all pressures $(P=.001)$. Longitudinal curvature was not significantly different from zero in the control case $(P=.2)$, but there was a small increase in longitudinal curvature in the patched state $(P=.001)$.

Regional strain. In Fig 6, we present data for first and second principal (major and minor) extensions in element 1 for a typical experiment $(a)$ and for all experiments $(b)$. Control results in Fig 6, $a$ indicate that major extensions increased progressively with inflation before patch implantation, whereas minor extensions remained relatively constant. There was little variation in the principal strains over the 9 gauss points in element 1 .

These individual data correspond closely to the average control results for all studies presented in Fig 6, $b$. Whereas the average major extension reached a maximum of $1.43 \pm 0.08$ at a pressure of $124 \pm 4.0 \mathrm{~mm} \mathrm{Hg}$ $(16.84 \pm 0.54 \mathrm{kPa})(P=.001)$, the average minor extension was not significant $(P=.9)$. The first and second principal extensions were aligned with circumferential and longitudinal directions, respectively, in all control studies.

Patch angioplasty altered the geometry of the aorta adjacent to the patch. The deformation associated with patch placement was characterized by estimating strain in the patched reference state relative to the control ref- erence state (Table II). There was a modest but significant circumferential extension, and the variation of longitudinal extension and circumferential-longitudinal shear between experiments was relatively large.

For the individual study presented in Fig 6, $a$, major extensions were increased relative to control for all pressures after patch implantation and there was a small increase in the minor extension with pressure. The widening of standard deviations indicates that patch angioplasty produced substantial regional variation of strain in the region adjacent to the patch. Average major extensions (Fig 6, $b$ ) rose to $1.55 \pm 0.08$ at a pressure of $122 \pm 4.0 \mathrm{~mm} \mathrm{Hg}(16.59 \pm 0.54 \mathrm{kPa})$ and were greater than paired control values at all pressures $(P=.001)$. There was substantial variation in the orientation of principal extensions after patch implantation, but major extensions tended to coincide with the circumferential direction, particularly as pressure increased.

Major extensions for all experiments at a pressure of $103 \mathrm{~mm} \mathrm{Hg}(14.0 \mathrm{kPa})$ are presented in Table III for each of the 9 gauss points in element 1 . Control principal extensions were relatively homogeneous across all gauss points. However, there was a consistent circumferential gradient in major extension in the aorta adjacent to the patch $(P=.001)$. The greatest change in major extension occurred at gauss point 6 adjacent to the center of the patch. Mean values of the major extension at this site were $1.40 \pm 0.08$ and $1.55 \pm 0.07$ for control and patched states, respectively, with the greatest individual difference from 1.25 to 1.53 . There was no systematic regional variation of minor extension, following patch implantation.

We were unable to demonstrate any consistent relationship between patch size (patch repair ratio), size of the aorta, and the incremental circumferential strain.

\section{Discussion}

This study was prompted by the hypothesis that synthetic patch repair of coarctation increases wall stress in 
Table III. Effect of patch implantation on major extensions
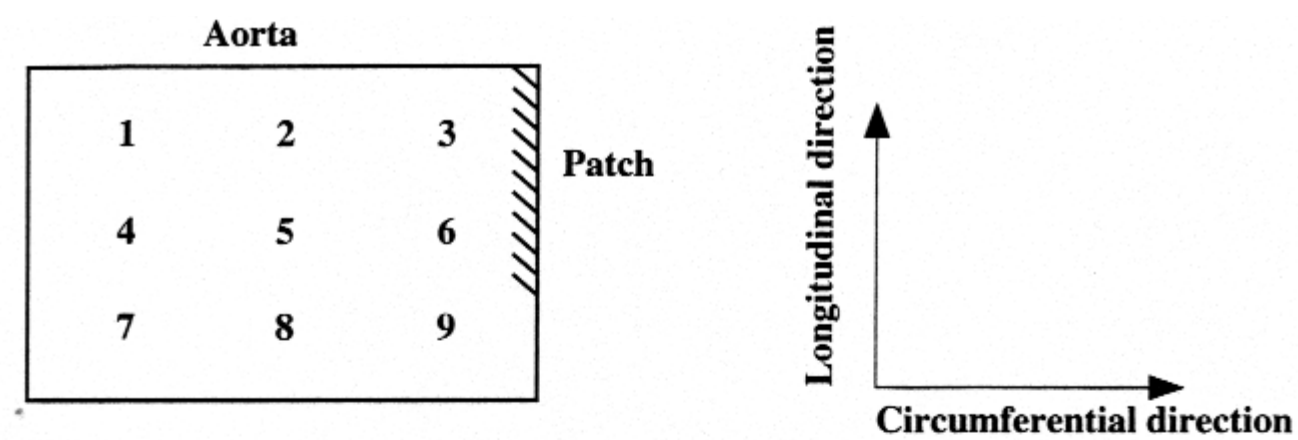

\begin{tabular}{|c|c|c|c|c|c|c|c|c|c|c|c|}
\hline Position & 1 & 2 & 3 & 4 & 5 & 6 & 7 & 8 & 9 & Mean & SD \\
\hline \multicolumn{2}{|c|}{ Aorta \# } & \multicolumn{6}{|c|}{ Control } & & & & \\
\hline 1 & 1.32 & 1.38 & 1.39 & 1.30 & 1.36 & 1.39 & 1.42 & 1.42 & 1.43 & 1.38 & 0.05 \\
\hline 2 & 1.45 & 1.45 & 1.46 & 1.41 & 1.43 & 1.44 & 1.39 & 1.41 & 1.41 & 1.43 & 0.02 \\
\hline 3 & 1.42 & 1.44 & 1.45 & 1.42 & 1.45 & 1.46 & 1.41 & 1.44 & 1.47 & 1.44 & 0.02 \\
\hline 4 & 1.36 & 1.36 & 1.43 & 1.37 & 1.38 & 1.36 & 1.37 & 1.36 & 1.32 & 1.37 & 0.03 \\
\hline 5 & 1.33 & 1.38 & 1.45 & 1.34 & 1.36 & 1.42 & 1.39 & 1.40 & 1.43 & 1.39 & 0.04 \\
\hline 6 & 1.52 & 1.50 & 1.51 & 1.48 & 1.49 & 1.48 & 1.45 & 1.42 & 1.41 & 1.47 & 0.04 \\
\hline 7 & 1.21 & 1.18 & 1.19 & 1.26 & 1.24 & 1.25 & 1.26 & 1.30 & 1.33 & 1.25 & 0.05 \\
\hline Mean & 1.37 & 1.39 & 1.41 & 1.37 & 1.39 & 1.40 & 1.39 & 1.39 & 1.40 & 1.39 & \\
\hline SD & 0.10 & 0.10 & 0.11 & 0.07 & 0.08 & 0.08 & 0.06 & 0.05 & 0.06 & 0.07 & \\
\hline
\end{tabular}

Aorta \#

After patch implantation

$\begin{array}{lllllllllllll}1 & 1.48 & 1.53 & 1.53 & 1.38 & 1.51 & 1.50 & 1.39 & 1.46 & 1.51 & 1.48 & 0.05\end{array}$

$\begin{array}{llllllllllll}2 & 1.54 & 1.54 & 1.65 & 1.55 & 1.53 & 1.59 & 1.48 & 1.49 & 1.50 & 1.54 & 0.05\end{array}$

$\begin{array}{lllllllllllll}3 & 1.49 & 1.58 & 1.60 & 1.50 & 1.59 & 1.61 & 1.50 & 1.55 & 1.60 & 1.56 & 0.05\end{array}$

$\begin{array}{llllllllllll}4 & 1.35 & 1.35 & 1.36 & 1.36 & 1.43 & 1.45 & 1.31 & 1.38 & 1.38 & 1.37 & 0.04\end{array}$

$\begin{array}{llllllllllll}5 & 1.46 & 1.50 & 1.53 & 1.48 & 1.52 & 1.50 & 1.47 & 1.48 & 1.45 & 1.49 & 0.03\end{array}$

$\begin{array}{llllllllllll}6 & 1.56 & 1.66 & 1.71 & 1.59 & 1.62 & 1.65 & 1.48 & 1.54 & 1.51 & 1.59 & 0.07\end{array}$

$\begin{array}{llllllllllll}7 & 1.15 & 1.27 & 1.35 & 1.36 & 1.43 & 1.53 & 1.30 & 1.36 & 1.23 & 1.33 & 0.11\end{array}$

$\begin{array}{lllllllllll}\text { Mean } & 1.43 & 1.49 & 1.53 & 1.46 & 1.52 & 1.55 & 1.42 & 1.47 & 1.46 & 1.48\end{array}$

$\begin{array}{llllllllllll}\text { SD } & 0.14 & 0.14 & 0.14 & 0.09 & 0.07 & 0.07 & 0.08 & 0.07 & 0.12 & 0.10\end{array}$

Control major extensions estimated at $103 \pm 3 \mathrm{~mm} \mathrm{Hg}(14.01 \pm .41 \mathrm{kPa})$

Major extensions after patch implantation estimated at $103 \pm 4 \mathrm{~mm} \mathrm{Hg}(14.01 \pm .54 \mathrm{kPa})$ 
the aorta adjacent to the patch. ${ }^{18}$ Abnormally high wall stress may cause elastin to fracture and degenerate, predisposing to attenuation of the aortic wall and subsequent true aneurysm formation. Coarctation is commonly associated with poststenotic dilatation of the aorta and aortic wall thinning. Moreover, coarctation may reflect a more widespread arterial disease because of the known association of coarctation and thoracic aortic aneurysm. ${ }^{21}$ Within this context, increased stress in the aortic wall around a coarctation repair is clearly undesirable and proof of the hypothesis would suggest that the operation of synthetic patch repair is flawed.

To address these issues, regional strain was estimated in human aortic wall before and after simulated coarctation repair by using a synthetic patch. Arterial geometry and deformation have been studied in vivo by using various techniques including angiography, ultrasonography, intravascular devices, and ultrasonic crystals. Although an in vivo model would have been desirable in the present study, none of these techniques could have provided regional strain data and an in vitro preparation was selected instead. The preparation used could not possibly simulate the exact geometry of a coarctation or the subsequent repair, but it was designed to determine strain fields around a patch in an abnormally large aorta, which is the inevitable consequence of repairing a coarctation by patch angioplasty.

The intrinsic resolution of 3-D coordinate reconstruction by using our biplane imaging system was on the order of $0.1 \mathrm{~mm}$. However, a number of factors combined to reduce the overall accuracy of coordinate reconstruction for marker points on the surface of the aorta. Surface marks were not always punctate and, in such cases, it was necessary to identify a specific feature of the mark and follow this through successive inflation steps. This sometimes became difficult as the mark itself deformed. It was also hard to identify the exact location of points in camera views where they appeared close to the boundary of the aorta.

The complexity of the finite element surface fitted to the reconstructed marker points was limited by the fact that the data points were bounded by a single element and by the smoothing constraints imposed in the fitting process. As a result, the fitted surfaces could not fully reproduce the variations in aortic geometry immediately adjacent to a synthetic patch. However, it is noteworthy that the average rms error for surface fits was $0.13 \mathrm{~mm}$, which compares well with the expected precision of 3-D coordinate reconstruction.

The estimates of surface curvature and regional strain obtained for the unpatched aorta confirm that it deforms as a cylinder with pressurization. Moreover,

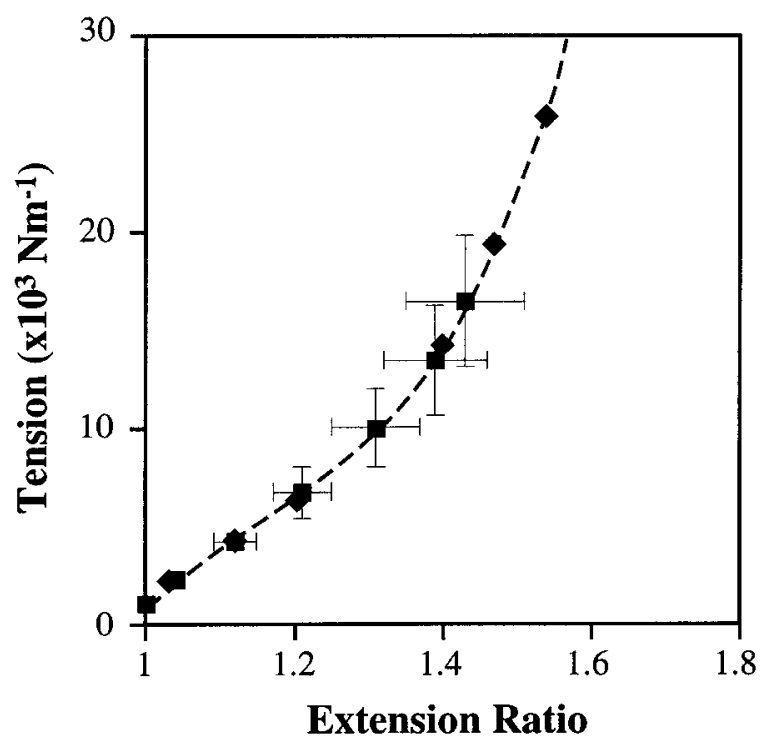

Fig 7. Circumferential tension as a function of circumferential extension ratio. Material relationship (dashed line) fitted to control data $(\square)$. Average circumferential extensions after patch implantation $(\diamond)$ are superimposed on the best-fit material relationship.

we have demonstrated that simulated coarctation repair with a synthetic patch substantially reduces circumferential curvature and slightly increases longitudinal curvature in the reference state. These changes in geometry were associated with complex patterns of deformation in the aorta immediately adjacent to the patch in the reference state.

The distribution of maximum extensions in the aorta adjacent to the patch is of considerable interest. Maximum extensions were greatest near the patch and progressively approached control values lateral to this. This result is not dependent on orientation of the patch with respect to the biplane measurement system because the side of the aorta on which the patch was viewed was randomly alternated. This result is also not due to the correction for initial strain after patch angioplasty, because regional extension gradients were, in general, greater for the uncorrected data.

The changes in major extensions adjacent to the patch appear to be relatively modest: an average $21 \%$ increase overall from 1.39 to 1.47 at a pressure of 103 $\mathrm{mm} \mathrm{Hg}(14.0 \mathrm{kPa})$, with a $35 \%$ increase in average extension from 1.40 to 1.54 at the gaussian quadrature point closest to the center of the patch. The largest individual principal extension (1.70 at $126 \mathrm{~mm} \mathrm{Hg} ; 17.14$ $\mathrm{kPa}$ ) was also observed in the aorta immediately adjacent to the patch. 
These data are placed in context if we consider the material properties of the aorta. Circumferential wall tension $\tau$ for a cylinder is given by $\tau=p_{t} r$, where $p_{t}$ is transmural pressure and $r$ is the radius of the aorta. For the control case, therefore, circumferential tension can be estimated from the data for curvature and transmural pressure to obtain the relationship between tension and extension presented in Fig 7. After simulated coarctation repair, the deformation of the aorta adjacent to the patch is initially noncylindrical and it is not possible to estimate circumferential tension by using the simple membrane solution above. However, it is reasonable to make inferences about the circumferential tensions associated with measured circumferential extensions from the material behavior of the aorta in the control state. The overall relationship between circumferential tension and extension ratio in Fig 7 is nonlinear with a progressive reduction in compliance for extensions greater than $30 \%$. These control data have been fitted with a third-order polynomial, which has been used to associate circumferential tensions with the regional circumferential extensions measured after patch angioplasty. There are a number of reasons why such an approach must be seen as qualitative rather than quantitative. First, longitudinal and shear deformations that occur in the patched state may well influence the circumferential material behavior of the aorta. Second, in the patched case, we are extrapolating beyond the range of extension ratios observed in the control state. However, the fact that the range of maximum extensions observed in the patched case was limited to $70 \%$ is consistent with the material relationship presented in Fig 7. It follows that the increases in circumferential extension observed in the patched case must be associated with disproportionate increases in circumferential tension and even greater increases in stress if the effects of wall thinning are taken into account.

An inherent problem with strain measurement in soft biologic tissues is the difficulty in defining an undeformed state to which strain measurements are referred. This certainly contributes to the substantial variation in the reported mechanical properties of aorta that have been obtained in other studies. ${ }^{19}$ There is significant residual stress in the aorta in vivo and when an aortic segment is removed from the body it retracts. In this study, aortic specimens were obtained at autopsy and in situ lengths were unknown. Because a standardized reference loading state was used in these experiments, it is possible to determine relative changes in regional strain in unpatched and patched specimens both within and between experiments. However, because of the variation in reference states used in other reported studies, it is not advisable to compare the absolute strains obtained here with those reported elsewhere in the literature. Instead, we have directly characterized the extent to which the aorta is operating on the nonlinear region of its stress-strain curve.

The latex balloon used to line the aorta before and after simulated patch repair did not appreciably influence the measured material properties of the aorta in this preparation. This method has previously been validated for the measurement of left ventricular intracavity volumes in small animals ${ }^{23}$ and negligible additional pressure was required to inflate the balloon.

The findings of this study demonstrate that regional strain in the aortic wall is significantly increased after simulated coarctation repair with a synthetic patch. These observations cannot be attributed to measurement error because the technique used is robust and well established. ${ }^{20}$ The increases in regional strain are associated with changes in local aortic wall geometry that are a direct result of patch implantation. The resultant flattening of the aorta in the circumferential direction must increase local stress and strain at equilibrium and it might be expected that the magnitude of these changes would be simply related to the relative circumferential dimensions of the patch and the aorta. In the present study, we were unable to establish any relationship between regional strain and patch size. This may be due to the complex geometry of the aorta in the region adjacent to the patch, but the observation that even a small patch may produce large increases in regional strain is noteworthy.

It is necessary to be circumspect in extrapolating from the findings of this acute in vitro study to the situation of a chronic patch aortoplasty. For instance, there will be ongoing tissue remodeling at the interface between patch and aorta after patch repair of coarctation. Scar tissue formation on the patch and the aorta immediately adjacent to it would be expected to relieve stress at the suture line and give rise to a more uniform strain distribution in the surrounding aortic wall than has been presented here. However, we have shown that strain is elevated throughout the aortic wall adjacent to the patch and that this is associated with circumferential flattening of the aorta due to patch implantation. It is unlikely that these geometric changes will be reversed by scar formation at the boundary of the patch. Turbulent flow, caused by changes in geometry at the site of repair, may also occur after patch aortoplasty. The rapid perturbations in mechanical loading due to turbulence are known to cause fragmentation and loss of elastin in blood vessels and this could contribute to aneurysm formation in the peripatch region. Finally, the current studies were carried out with patch- 
es of Dacron graft, but a variety of other materials including polytetrafluoroethylene* and glutaraldehydefixed bovine pericardium are also used for coarctation repair. We believe that our experimental model is representative within this context, because Dacron, polytetrafluoroethylene, and glutaraldehyde-fixed bovine pericardium are all highly inextensible at systemic pressure. However, a realistic finite element computer model is seen as the appropriate platform for systematic analysis of the effects of patch geometry, dimensions and material properties on the distribution of stress and strain in the surrounding aortic wall. The present study provides the data necessary to implement and validate such a model and we will use this approach in the future to extend this work.

In conclusion, the findings of this study suggest that synthetic patch repair of coarctation of the aorta may produce increases in wall strain (and disproportionate increases in stress) adjacent to the patch. The unpredictable appearance of true aneurysms in patients after coarctation repair by this technique may reflect the variability of regional strain in the aortic wall, which, even with a small synthetic patch, may be substantial. Because of this unfavorable biomechanical feature, synthetic patch repair of coarctation should not be preferentially used.

We are grateful for the help of Mrs Jane Owenby (UAB) with manuscript preparation.

\section{REFERENCES}

1. Vosschulte K. Isthmusplastic zur Behandlung der aorten Isthmusstenose. Thoraxchirurgie 1956;4:443-50.

2. Olsson P, Soderlund S, Dubiel WT, Ovenfors CO. Patch grafts or tubular grafts in the repair of coarctation of the aorta. Scand $\mathbf{J}$ Thorac Cardiovasc Surg 1976;10:139-43.

3. Bergdahl L, Ljungqvist A. Long-term results after repair of coarctation of the aorta by patch grafting. J Thorac Cardiovasc Surg 1980;80:177-81.

4. Ala-Kulju K, Jarvinen A, Maamies T, Mattila S, Merikalio E. Late aneurysms after patch aortoplasty for coarctation of the aorta in adults. Thorac Cardiovasc Surg 1983;31:301-5.

5. Clarkson PM, Brandt PWT, Barratt-Boyes BG, Rutherford JD, Kerr AR, Neutze JM. Prosthetic repair of coarctation of the aorta with particular reference to Dacron onlay patch grafts and late aneurysm formation. Am J Cardiol 1985;56:342-6.

6. del Nido PJ, Williams WG, Wilson GJ, Coles JG, Moes CA, Hosokawa Y, et al. Synthetic patch angioplasty for repair of

${ }^{*}$ Gore-Tex patch, registered trademark of W. L. Gore \& Associates, Inc, Flagstaff, Ariz. coarctation of the aorta: experience with aneurysm formation Circulation 1986;74(3 Pt 2):I-32-6.

7. Rheuban KS, Gutgesell HP, Carpenter MA, Jedeikin R, Damman $\mathrm{JF}$, Kron IL, et al. Aortic aneurysm after patch angioplasty for aortic isthmic coarctation in childhood. Am J Cardiol 1986;58:178-80.

8. Hehrlein FW, Mulch R, Rautenburg HW, Schlepper M, Scheld $\mathrm{HH}$. Incidence and pathogenesis of late aneurysms after patch graft aortoplasty for coarctation. J Thorac Cardiovasc Surg 1986;92:226-30

9. DeSanto A, Bills RG, King H, Waller B, Brown JW. Pathogenesis of aneurysm formation opposite prosthetic patches used for coarctation repair. J Thorac Cardiovasc Surg 1987;94:720-3.

10. McGoldrick JP, Brown IW, Ross DN. Coarctation of aorta: late aneurysm formation with Dacron onlay patch grafting. Ann Thorac Surg 1988;45:89-90.

11. Ala-Kulju K, Keikkinen L. Aneurysms after patch graft aortoplasty for coarctation of the aorta: long-term results of surgical management. Ann Thorac Surg 1989;47:853-6.

12. Bromberg BI, Beekman RH, Rocchini AP, Snider AR, Bank ER, Heidelberger K, et al. Aortic aneurysm after patch aortoplasty repair of coarctation: a prospective analysis of prevalence, screening tests and risk. J Am Coll Cardiol 1989;14:734-41.

13. John CN, Cartmill TB, Johnson DC, Nunn GR. Report of four cases of aneurysm complicating patch aortoplasty for repair of coarctation of the aorta. Aust N Z J Surg 1989;59:748-50.

14. Mendolsohn AM, Crowley DC, Lindauer A, Beekman RH. Rapid progression of aortic aneurysms after patch aortoplasty repair of the aorta. J Am Coll Cardiol 1992;20:381-5.

15. Aebert H, Laas J, Bednarski P, Koch U, Prokop M, Borst HG. High incidence of aneurysm formation following patch plasty repair of coarctation. Eur J Cardiothorac Surg 1993;7:200-5.

16. Parks WJ, Thang DN, Plauth WH, Bank ER, Sheppard SK, Pettigrew RI, et al. Incidence of aneurysm formation after Dacron patch aortoplasty repair for coarctation of the aorta: long-term results and assessment utilizing magnetic resonance angiography with three-dimensional rendering. J Am Coll Cardiol 1995;26:266-71.

17. Knyshov GV, Sitar LL, Glagola MD, Alamanyuk MY. Aortic aneurysms at the site of repair of coarctation of the aorta: a review of 48 patients. Ann Thorac Surg 1996;61:935-9.

18. McGiffin DC, McGiffin PB, Galbraith AJ, Cross RB. Aortic wall stress profile after repair of coarctation of the aorta: Is it related to subsequent true aneurysm formtion? J Thorac Cardiovasc Surg 1992;104:924-31.

19. Bergel DH. The visco-elastic properties of the arterial wall. Thesis for $\mathrm{PhD}$, University of London, 1960.

20. McCulloch AD, Smaill BH, Hunter PJ. Left ventricular epicardial deformation in isolated arrested dog heart. Am J Physiol 1987;252:H233-41.

21. Young AA, Orr R, Smaill BH, Dell'Italia LJ. Three-dimensional changes in left and right ventricular geometry in chronic mitral regurgitation. Am J Physiol 1996;271:H2689-700.

22. Skandalakis JE, Edwards BF, Gray SW, Davis BM, Hopkins WA. Coarctation of the aorta with aneurysm. Surg Gynecol Obstet 1960;111:307-26.

23. Suga H, Sagawa K. Accuracy of ventricular lumen volume measurement by intraventricular balloon method. Am J Physiol 1979;236:H506-7. 